\title{
Mullerian duct anomalies presenting with primary amenorrhoea
}

\author{
Piyusha Chandrayan*, Usha Parekh, Niyati Jain
}

Department of obstetrics \& gynaecology, SBKS Medical Institute and Research Centre, Pipariya, Waghodiya, Vadodara 391760, Gujarat, India

Received: 13 October 2015

Revised: 23 December 2016

Accepted: 04 January 2016

*Correspondence:

Dr. Piyusha Chandrayan,

E-mail: piyusha011@gmail.com

Copyright: $(\subset)$ the author(s), publisher and licensee Medip Academy. This is an open-access article distributed under the terms of the Creative Commons Attribution Non-Commercial License, which permits unrestricted non-commercial use, distribution, and reproduction in any medium, provided the original work is properly cited.

\begin{abstract}
Background: Primary amenorrhoea is defined as the absence of menstruation by 14 years of age when there is no visible development of secondary sexual characteristics or by 16 years of age in the presence of normal secondary sexual characteristics. Primary Amenorrhoea occurs if there is a blockage of the outflow tract, or the outflow tract is missing, or there is no functioning uterus. The causes include: Transverse blockages, Mullerian Anomalies, and absence of functioning endometrium. The true incidence of obstructive Müllerian anomalies is unknown, but is believed to be between $0.1 \%$ and $3.8 \%$. The second most common cause of primary amenorrhoea is mullerian anomaly. The aim of this study is to find the most common cause of primary amenorrhoea among mullerian anomalies and its renal association, the presenting symptom and the modalities to investigate it.

Methods: After obtaining approval from the ethical committee, a retrospective study was undertaken of all cases of primary amenorrhoea presented at Dhiraj General Hospital, affiliated to SBKS Medical college and research centre, Waghodiya during a 5 year period (June 2011-June 2015). In inclusion criteria, all non-pregnant women of Reproductive age group attending Gynaecology O.P.D. at Dhiraj General Hospital with complains of primary amenorrhoea with normal secondary sex characters were included in the study (June 2011-June 2015). In exclusion criteria, after the detailed investigations, all non-pregnant women with complain of primary amenorrhoea due to any other cause than mullerian anomaly were excluded.

Results: In this study, most of the patients belonged to Class I, i.e. these patients had absent uterus, cervix and fallopian tubes along with upper part of vagina(61.5\%) consisting of MRKH type 1 . Nearly, $31 \%$ belonged to class II type; these patients had transverse vaginal septum with or without uterine anomaly. One patient belonged to class III type, $(7.6 \%)$ i.e. imperforate hymen with hematocolpos and hematometra.

Conclusions: We conclude that Mayer-Rokitansky-Kuster-Hauser syndrome (MRKH) with normal female secondary sexual development is the commonest cause of primary amenorrhea in our study.
\end{abstract}

Keywords: Primary amenorrhoea, MRKH syndrome, Mullerian duct, Secondary sexual characters, Renal anomaly, MRI

\section{INTRODUCTION}

Primary amenorrhoea is defined as the absence of menstruation by 14 years of age when there is no visible development of secondary sexual characteristics or by 16 years of age in the presence of normal secondary sexual characteristics. ${ }^{1}$

The female reproductive tract develops from a pair of mullerian ducts that form the: fallopian tube, uterus, cervix, and the upper two-third of vagina .Primary 
Amenorrhoea occurs if there is a blockage of the outflow tract, or the outflow tract is missing, or there is no functioning uterus. The causes include: Transverse blockages, Mullerian Anomalies, and absence of functioning endometrium. ${ }^{2}$

The defect results from interruption or dysregulation in mullerian duct development at various stages of morphogenesis. This may be due to intrauterine and extra uterine elements, genetic causes or due to teratogenic drugs and may run in families or may occur sporadically. ${ }^{3}$

Other modes of inheritance include autosomal dominant, autosomal recessive, and $\mathrm{X}$ linked disorders. These may also result as multiple malformation syndrome. ${ }^{4}$ The existence of mullerian defects dates back to antiquity, around $300 \mathrm{BC}$. Colombo reported the first documented case of vaginal agenesis (uterus and vagina) in the 16th century. ${ }^{2}$

The true incidence of obstructive Müllerian anomalies is unknown, but is believed to be between $0.1 \%$ and $3.8 \%$.The second most common cause of primary amenorrhoea is mullerian anomaly. ${ }^{8}$

The aim of this study is to find the most common cause of primary amenorrhoea among mullerian anomalies and its renal association, the presenting symptom and the modalities to investigate it.

\section{METHODS}

After obtaining approval from the ethical committee, a retrospective study was undertaken of all cases of primary amenorrhoea presented at Dhiraj General Hospital, affiliated to SBKS Medical college and research centre, Waghodiya during a 5 year period (June2011June2015).

\section{Inclusion criteria}

All non-pregnant women of Reproductive age group attending Gynaecology O.P.D. at Dhiraj General Hospital with complains of primary amenorrhoea with normal secondary sex characters were included in the study. (June2011-June2015).

\section{Exclusion criteria}

After the detailed investigations, all non-pregnant women with complains of primary amenorrhoea due to any other cause than mullerian anomaly were excluded.

In the study, detailed relevant family history to rule out genetic causes, sexual history stressing difficulty in conjugation of marriage, psychological built of the woman (male/female) was taken followed by routine clinical examination \&investigations.
Physical examination focused on nutritional status, secondary sexual characters, per abdomen, local hymen examination, per vaginal and per rectal examination to determine the cause of Primary Amenorrhoea.

RBS, S.LH, S.FSH, S. Prolactin and S. TSH were done as part of the protocol for primary amenorrhoea evaluation.

On the basis of MRI, diagnosis was made. In all cases, further evaluation was done if needed by IVP to find out renal anomalies which are commonly associated with congenital mullerian anomalies. Urologists were consulted to manage the associated renal anomalies, if any.

Cytogenetic studies was not a part of the routine protocol of primary amenorrhoea as most of the patients were non affording and the institute does not have on site facility for this test.

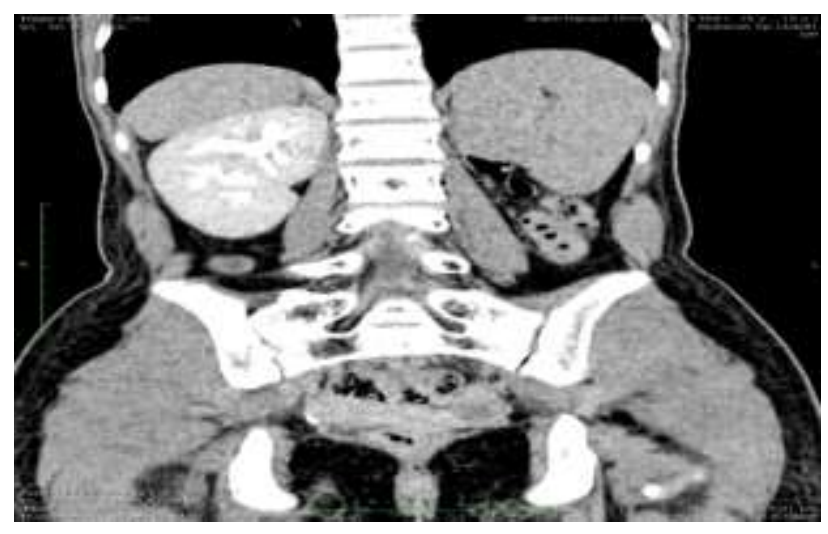

Figure 1: IVP, of a 15 year old patient showing single kidney with bicorn ate uterus.

\section{RESULTS}

Table 1: Age distribution.

\begin{tabular}{|lllll|}
\hline $\begin{array}{l}\text { Age } \\
\text { distribution }\end{array}$ & Number=13 & Percentage & $\begin{array}{l}\text { Marital } \\
\text { status }\end{array}$ & Percentage \\
\hline 13-15yrs & 2 & 15 & 0 & 0 \\
\hline 16-18yrs & 6 & 47 & 1 & 7.6 \\
\hline $19-21 y r s$ & 5 & 38 & 5 & 38.4 \\
\hline
\end{tabular}

Table 2: Presenting symptoms.

\begin{tabular}{|c|c|c|}
\hline Symptoms & Number $=13$ & Percentage \\
\hline Only primary amenorrhoea & 4 & 31 \\
\hline $\begin{array}{l}\text { Primary amenorrhoea with pain } \\
\text { in abdomen }\end{array}$ & n & 23 \\
\hline $\begin{array}{l}\text { Primary amenorrhoea with } \\
\text { Difficulty in conjugation of } \\
\text { marriage }\end{array}$ & 4 & 31 \\
\hline $\begin{array}{l}\text { Primary amenorrhoea with } \\
\text { retention of urine }\end{array}$ & 1 & 7.6 \\
\hline $\begin{array}{l}\text { Primary amenorrhoea with } \\
\text { primary infertility }\end{array}$ & 1 & 7.6 \\
\hline
\end{tabular}


Table 3: On per vaginum clinical examination.

\begin{tabular}{|lll|}
\hline Findings & Number=5 & Percentage \\
\hline $\begin{array}{l}\text { Vagina as blind } \\
\text { pouch/vagina as a dimple }\end{array}$ & 2 & 15.3 \\
\hline Imperforate hymen & 1 & 7.6 \\
\hline Transverse vaginal septum & 2 & 15.3 \\
\hline
\end{tabular}

Table 4: MRI \& type of mullerian agenesis diagnosed.

\begin{tabular}{|lcc|}
\hline $\begin{array}{l}\text { Anomalies of } \\
\text { mullerian duct }\end{array}$ & Pumber= 13 & Percentage \\
\hline $\begin{array}{l}\text { Dysgenesis of mullerian } \\
\text { duct (Class I) }\end{array}$ & 8 & 61.5 \\
\hline $\begin{array}{l}\text { Disorders of vertical fusion } \\
\text { of the mullerian duct } \\
\text { (Class II) }\end{array}$ & 4 & 30.7 \\
\hline $\begin{array}{l}\text { Disorders of lateral fusion } \\
\text { of mullerian duct (Class }\end{array}$ & 1 & 7.6 \\
III) & - & - \\
\hline $\begin{array}{l}\text { Unusual configuration of } \\
\text { vertical-lateral fusion } \\
\text { defects (Class IV) }\end{array}$ & - & \\
\hline
\end{tabular}

Table 5: Renal anomaly association with mullerian duct anomaly.

\begin{tabular}{|llll|}
\hline Anomalies & $\begin{array}{l}\text { Associated } \\
\text { with } \\
\text { mullerian } \\
\text { anomaly }\end{array}$ & $\begin{array}{l}\text { Associated } \\
\text { renal } \\
\text { anomaly }\end{array}$ & $\begin{array}{l}\text { \% of renal } \\
\text { Association }\end{array}$ \\
\hline Class I & 8 & 6 & 75 \\
\hline Class II & 4 & 4 & 100 \\
\hline Class III & - & - & - \\
\hline Class IV & - & - & - \\
\hline Total & 12 & 10 & 100 \\
\hline
\end{tabular}

During this period total number of patients attending the gynaecology outpatient department was 19,800. We had 13 patients who presented with primary amenorrhea. The incidence of primary amenorrhea in our institute was $0.65 \%$ which is very low.

1. In our study, the most common age group with findings of mullerian anomaly was 16-18 years, comprising $47 \%$, whereas $19-21$ years was $38 \%$ and $13-15$ years, $15 \%$.

2. Primarily all patients presented with common complaint of primary amenorrhoea. Patients also presented with associated symptoms like pain in abdomen (23\%), difficulty in conjugation of marriage $(31 \%)$, primary infertility $(7.6 \%)$, and retention of urine $(7.6 \%)$.

3. 3. Most of these patients, on per vaginal examination gave significant information(apx.38\%) like vagina as a blind pouch or like a dimple $(15.3 \%)$, presence of the transverse vaginal septum though rare6( $15.3 \%) \&$ imperforated hymen, $(7.6 \%)$.

4. In this study, most of the patients belonged to Class I, i.e. these patients had absent uterus, cervix and fallopian tubes along with upper part of vagina $(61.5 \%)$ consisting of MRKH type 1 . Nearly, $31 \%$ belonged to class II type; these patients had transverse vaginal septum with or without uterine anomaly. One patient belonged to class III type, $(7.6 \%)$ i.e. imperforate hymen with hematocolpos and hematometra.

5. In the study, the renal anomaly was associated with all Class II mullerian anomaly (100\%) whereas Class I had $75 \%$ association with renal anomaly. Other classes didn't have any associated renal anomaly.

6. In our set up MRI was performed in all patients which helped in diagnosing the type of mullerian anomaly along with the associated renal anomaly. Additional IVP was not needed.

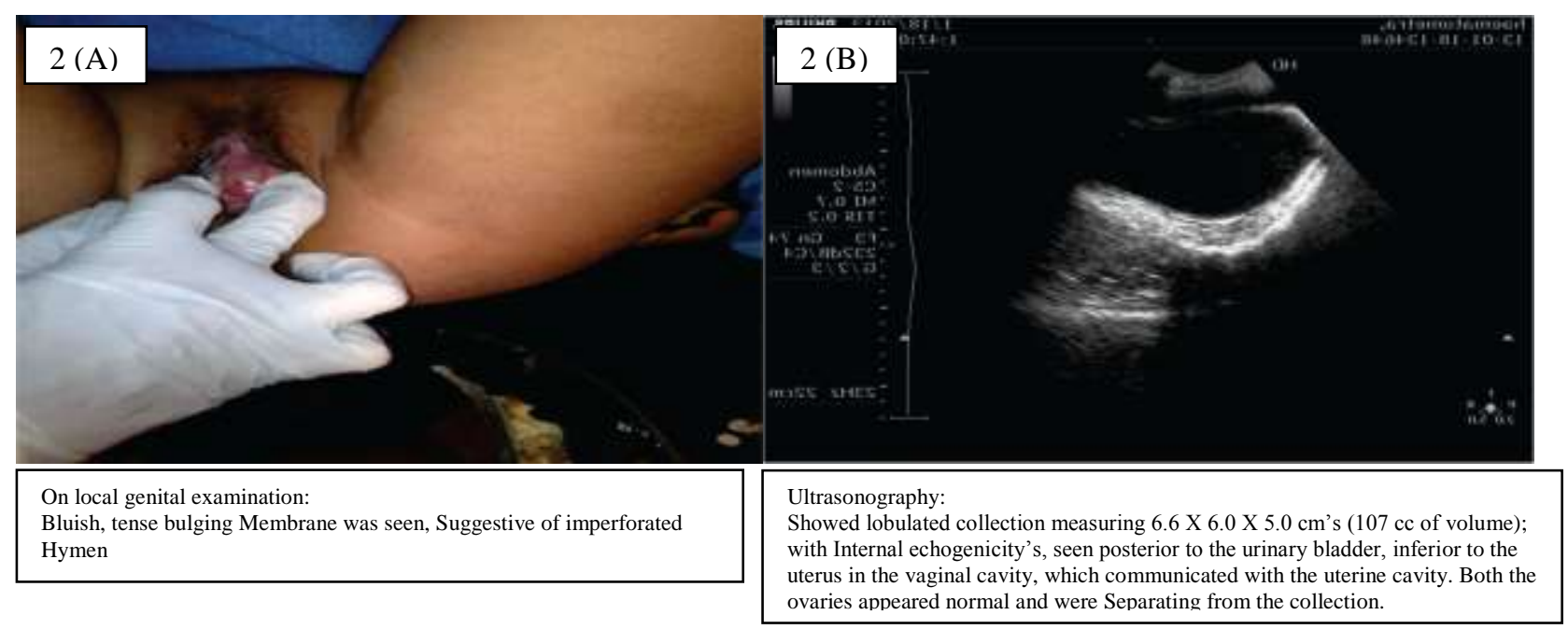

Figure 2: Imperforate hymen. 


\section{DISCUSSION}

The fusion of two mullerian ducts (paramesonephric ducts) forms the upper two thirds of the vagina, as well as the cervix and uterus. Embryonically, the female reproductive tract develops from the genital ducts and urogenital sinus. The mullerian ducts grow downwards and extend to enter the urogenital sinus, after which they get fused. Uterine cavitation occurs at site of fused mullerian ducts and the uterus continues to grow downward. Cavitation is complete and the lower segment and cervix, along with the upper part of vagina are formed. $^{7}$

In this study we have restricted ourselves to primary amenorrhoea with normal secondary sexual characteristics solely caused by mullerian anomalies.

The American Fertility Society has given the following classification of Mullerian Anomaly on the basis of failure of normal development.

I. Dysgenesis of the mullerian ducts. Ex. MayerRokitansky-Kuster-Hauser syndrome

II. Disorders of vertical fusion of the mullerian ducts

a. Transverse vaginal septum

b. Cervical agenesis or dysgenesis

III. Disorders of the lateral fusion of the mullerian ducts

a. Asymmetric obstructed disorder of uterus or vagina usually associated with ipsilateral renal agenesis

- Unicornuate uterus with a non-communicating rudimentary horn

- Unilateral obstruction of a cavity of a double uterus

- Unilateral vaginal obstruction associated with double uterus

b. Symmetric- unobstructed

- Didelphis uterus

- Septate uterus

- Bicorn ate uterus

- $\mathrm{T}$ shaped uterine cavity(diethylstilboestrol related)

- Unicornuate uterus with or without a rudimentary horn

IV. Unusual configuration of vertical-lateral fusion defects.

Anomalies are grouped according to similarities of clinical manifestations, treatment and prognosis for fetal salvage.

- In the study, the most prevalent age group manifesting with complaint of primary amenorrhoea belonged to the adolescent group (16-18yrs), comprising $47 \%$ of study. The incidence of mullerian anomaly as per this study is $0.67 \%$.
According to ASOG, the incidence and prevalence of mullerian duct anomaly is still unknown. ${ }^{11}$ As per Orphanet Journal of rare diseases, 2007: The incidence of MRKH syndrome has been estimated as 1 in 4500 female births.

- Some of the patients with chief complaint of primary amenorrhoea had associated complaints like - pain in lower abdomen (23\%), or some with difficulty in sexual activity \& infertility (31\%) are included, while a few patients in higher age group also had only primary infertility $(7.6 \%)$ and retention of urine $(7.6 \%)$.

Clinical Obstetrics \& Gynaecology, by Shulman et al, 2008 published an article stating clinicians should be suspicious for Müllerian anomalies in cases of primary amenorrhea, pelvic pain, repetitive pregnancy loss, and certain adverse obstetrical outcomes. ${ }^{15}$

- On Clinical Examination, vagina was felt as a blind pouch or dimple in $15.3 \%$, transverse vaginal septum was present in $15.3 \%$ and imperforate hymen in $7.6 \%$. The per vaginum examination was only carried out in married females constituting $46 \%$ whereas unmarried females, nearly $54 \%$ were admitted and after taking informed consent diagnostic laparoscopy was carried out.

Peter et al; Examined the clinical aspect of MRKH syndrome, according to this, the typical form was diagnosed in 25 patients $(47 \%)$, the atypical form in 11 patients (21\%), and the most marked form-the MURCS type-in 17 patients (32\%). Associated malformations were notably frequent among the patients. ${ }^{14}$

- In this study, the most common cause of primary amenorrhoea belonged to Class I, MayerRokitansky-Kuster-Hauser (MRKH) Syndrome. It is characterized by congenital aplasia of uterus and upper $2 / 3$ rd part of the vagina in women showing normal development of secondary sexual characteristics and a normal 46, XX karyotype. Type I (isolated) MRKH is less frequent than MURCS association. As per our study, type I that is Dysgenesis of mullerian duct or MRKH is most common and nearly $75 \%$ is associated with renal anomaly.

Class II type of mullerian anomaly constituted $30.7 \%$ of patients. They had uterine or cervical anomaly with transverse vaginal septum. Though, the exact etiology of transverse vaginal septum is unknown but its incidence has been reported to be 2 in 100,000 female live births making it one of the rarest anomalies of the female genital tract. $^{6}$ These patients had associated renal anomaly like presence of single kidney. The septum was 
resected and hematometra if present was drained as a treatment modality.

Class III, accounted for $15.3 \%$ of the patients. This young girl typically presented with imperforate hymen with hematometra and hematocolpos. The hymen was incised and hematocolpos was drained followed by spontaneous drainage of hematometra in head up position for few days.

As per a classification of female genital tract congenital anomalies by A. Di Spiezio Sardo et al, the uterus was the organ most frequently involved (30/39: 76.9\%), followed by cervix (26/39: 66.7\%) and vagina (23/39: $59 \%$ ). The congenital anomaly involved a single organ in 12 types of anomalies (12/39: 30.8\%), while multiple organs and/or segments of Müllerian ducts in more than one stages of embryologic development (complex anomalies) were simultaneously affected in 27 types of anomalies (27/39: 69.2\%).

- Most commonly associated renal anomaly was found with class II type (100\%). These patients had presence of ipsilateral kidney. In class I with nearly $75 \%$ association of renal anomaly, presence of single kidney in $15 \%$, horse-shoe shaped kidney in $25 \%$ \& ectopic kidney with ectopic ureter $35 \%$.was found.

$\mathrm{Li}$, Saying et al in his analysis of Association of Renal Agenesis and Mullerian Duct Anomalies concluded that renal agenesis is more commonly seen in uterus didelphys, i.e. type II than in other types of MDAs. Renal agenesis in patients with uterus didelphys is often ipsilateral to an obstructing, transverse, hemivaginal septum. $^{16}$

A study by Thomas Jefferson University Hosp, Philadelphia, 2013 showed that traditional procedures like combined hysteroscopy/laparoscopy, HSG were not only uncomfortable \& invasive but HSG also posed risk of radiation exposure. Thus, MRI became the gold standard diagnostic imaging modality but entailing cost, patient discomfort and inconvenience. ${ }^{17, \mathrm{I}}$

In our study, MRI is the gold standard of diagnosis and so it was performed in all our patients who presented with primary amenorrhoea.

The patients with blind vaginal pouch, who were married or about to get married $46 \%$, underwent vaginoplasty as regular coitus will not allow obliteration of surgically created space. Repair before puberty is associated with a high rate of vaginal stenosis, and re-repair with vaginal reconstruction may be required later for adequate menstruation and coital function. Hence, those belonging to too young age group, 13-15 years and (15\%) were called later for correction.

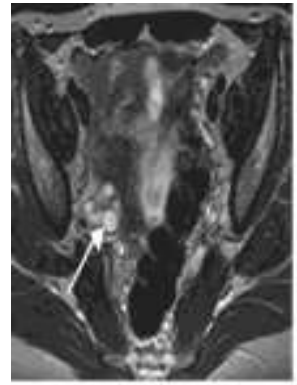

(a)

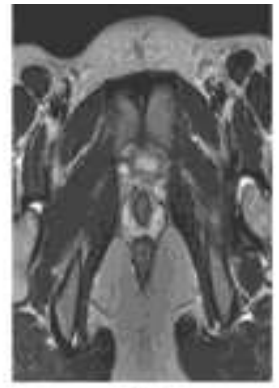

(b)

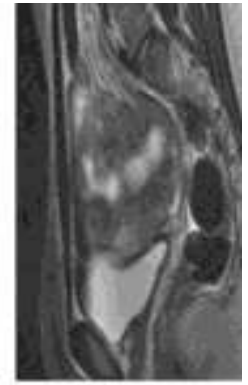

Figure 3: MRI of a pelvis in 20 year-old women with uterine and vaginal agenesis (Class I). (a, b) Axial and (c) sagittal $\mathbf{T}$ demonstrates normal ovaries (arrow depicts the right ovary; left not shown) and absent uterus and upper vagina.

\section{CONCLUSIONS}

- We conclude that Mayer-Rokitansky-KusterHauser syndrome (MRKH) with normal female secondary sexual development is the commonest cause of primary amenorrhea in our study.

- Mullerian anomaly usually remains undetected until the patient presents with primary amenorrhea or difficulty in conjugation of marriage.

- Magnetic resonance imaging is currently the gold standard for diagnosis of Mullerian Duct anomalies.

- MRKH syndrome generates anxiety and psychological distress affecting patient's quality of life, thus requiring a multidisciplinary approach, counselling with the support groups, and constant family support.

- The indicated therapeutic treatment is the surgical or non-surgical creation of a neovagina, which may allow these patients to have a normal sex life.

- Presence of functional ovaries in these women allows the production of normal ovules. Hence, patients desirous of having children should be encouraged to adopt, or assisted reproduction techniques to have biological children should be suggested.

Funding: No funding sources

Conflict of interest: None declared

Ethical approval: The study was approved by the Institutional Ethics Committee

\section{REFERENCES}

1. Folch M, I Pigem, Konje JC. Müllerian agenesis: etiology, diagnosis, and management. Obstetrical \& gynecological survey 55.10. 2000:644-9.

2. LeRoy, Suzanne. Vaginal reconstruction in adolescent females with Mayer-Rokitansky-KusterHauser syndrome. Plastic Surgical Nursing 21.1. 2001:23-7. 
3. Krishna D, Bains R. Reconstructive surgical management of cryptomenorrhoea because of complete vaginal agenesis. Open Journal of Obstetrics and Gynecology 1.04. 2011:243.

4. Rajani PM. Mullerian anomalies: A cause of primary amenorrhea." International J Reproduction Contraception Obstet Gynaecol 2.3. 2013:393.

5. Chandler TM. Müllerian duct anomalies: from diagnosis to intervention. The British journal of radiology. 2014.

6. Deligeoroglou E. Management of hematocolpos in adolescents with transverse vaginal septum. Archives of gynecology and obstetrics 285.4. 2012:1083-7.

7. American Journal of Medical Genetics. 2000;94,(1):9-12.

8. Speroff L, Fritz MA. Clinical Gynaecologic Endocrinology and Infertility. Lippincott, Williams \& Wilkins. 2005:403.

9. Rock JA, Lesley L. Breech. Te linde's operative gynaecology, tenth edition. Surgery for Anomalies of the Mullerian Ducts. 2010:539-41.

10. Parikh R, Nakum K, Kadikar G, Gokhle A. Mullerian anomalies: A cause of primary amenorrhea. International Journal of Reproduction, Contraception, Obstetrics and Gynecology.
2013;2,(3):393.

doi:10.5455/23201770.ijrcog20130926.

11. Gaetano T, Corrado D, Basso C. Orphanet Journal of Rare. Orphanet journal of rare diseases. 2007:45.

12. Oppelt, Peter. Clinical aspects of MayerRokitansky-Kuester-Hauser syndrome: recommendations for clinical diagnosis and staging. Human Reproduction 21.3. 2006:792-97.

13. Clinical Obstetrics \&Gynaecology; Paediatric and Adolescent Gynaecology. 2008;51,(2):214-22. doi: 10.1097/GRF.0b013e31816feb

14. Li Saying. Association of renal agenesis and mullerian duct anomalies. Journal of computer assisted tomography 24.6. 2000:829-34.

15. Article on Diagnostic Modalities for mullerian anomalies. Dept of obstetric \& gynaecology, Thomas Jefferson University Hospital, Philadelphia PA. 2013;21(3).

16. Marcal, Leonardo. Mullerian duct anomalies: MR imaging. Abdominal imaging 36.6. 2011:756-64.

Cite this article as: Chandrayan P, Parekh U, Jain N. Mullerian duct anomalies presenting with primary amenorrhoea. Int J Reprod Contracept Obstet Gynecol 2016;5:300-05. 